\title{
Composition and functional analysis of low-molecular-weight glutenin alleles with Aroona near-isogenic lines of bread wheat
}

\author{
Xiaofei Zhang ${ }^{1,2+}$, Hui Jin ${ }^{1 \dagger}$, Yan Zhang ${ }^{1}$, Dongcheng Liư ${ }^{2}$, Genying Li ${ }^{3}$, Xianchun Xia ${ }^{1}$, Zhonghu He ${ }^{1,4^{*}}$
} and Aimin Zhang ${ }^{2^{*}}$

\begin{abstract}
Background: Low-molecular-weight glutenin subunits (LMW-GS) strongly influence the bread-making quality of bread wheat. These proteins are encoded by a multi-gene family located at the G/u-A3, Glu-B3 and Glu-D3 loci on the short arms of homoeologous group 1 chromosomes, and show high allelic variation. To characterize the genetic and protein compositions of LMW-GS alleles, we investigated 16 Aroona near-isogenic lines (NILs) using SDS-PAGE, 2D-PAGE and the LMW-GS gene marker system. Moreover, the composition of glutenin macro-polymers, dough properties and pan bread quality parameters were determined for functional analysis of LMW-GS alleles in the NILS.

Results: Using the LMW-GS gene marker system, 14-20 LMW-GS genes were identified in individual NILs. At the Glu-A3 locus, two m-type and 2-4 i-type genes were identified and their allelic variants showed high polymorphisms in length and nucleotide sequences. The Glu-A3d allele possessed three active genes, the highest number among Glu-A3 alleles. At the Glu-B3 locus, 2-3 m-type and 1-3 s-type genes were identified from individual NILs. Based on the different compositions of s-type genes, Glu-B3 alleles were divided into two groups, one containing Glu-B3a, B3b, B3f and B3g, and the other comprising Glu-B3C,B3d, B3h and B3i. Eight conserved genes were identified among Glu-D3 alleles, except for Glu-D3f. The protein products of the unique active genes in each NIL were detected using protein electrophoresis. Among Glu-3 alleles, the Glu-A3e genotype without i-type LMW-GS performed worst in almost all quality properties. Glu-B3b, B3g and B3i showed better quality parameters than the other Glu-B3 alleles, whereas the Glu-B3C allele containing s-type genes with low expression levels had an inferior effect on bread-making quality. Due to the conserved genes at Glu-D3 locus, Glu-D3 alleles showed no significant differences in effects on all quality parameters.

(Continued on next page)
\end{abstract}

\footnotetext{
*Correspondence: zhhecaas@163.com; amzhang@genetics.ac.cn

${ }^{\dagger}$ Equal contributors

${ }^{1}$ Institute of Crop Science, National Wheat Improvement Center, Chinese Academy of Agricultural Sciences (CAAS), 12 Zhongguancun South Street, Beijing 100081, China

${ }^{2}$ State Key Laboratory of Plant Cell and Chromosome Engineering, National Center for Plant Gene Research, Institute of Genetics and Developmental Biology, Chinese Academy of Sciences, 1 West Beichen Road, Beijing 100101, China

Full list of author information is available at the end of the article
}

\section{Biomed Central}

(c) 2012 Zhang et al.; licensee BioMed Central Ltd. This is an Open Access article distributed under the terms of the Creative Commons Attribution License (http://creativecommons.org/licenses/by/2.0), which permits unrestricted use, distribution, and reproduction in any medium, provided the original work is properly cited. 
(Continued from previous page)

Conclusions: This work provided new insights into the composition and function of 18 LMW-GS alleles in bread wheat. The variation of i-type genes mainly contributed to the high diversity of Glu-A3 alleles, and the differences among Glu-B3 alleles were mainly derived from the high polymorphism of s-type genes. Among LMW-GS alleles, Glu-A3e and Glu-B3C represented inferior alleles for bread-making quality, whereas Glu-A3d, Glu-B3b, Glu-B3g and Glu-B3i were correlated with superior bread-making quality. Glu-D3 alleles played minor roles in determining quality variation in bread wheat. Thus, LMW-GS alleles not only affect dough extensibility but greatly contribute to the dough resistance, glutenin macro-polymers and bread quality.

Keywords: Triticum aestivum, Glu-3 alleles, Bread-making quality

\section{Background}

The unique viscoelastic properties conferred by gluten proteins in bread wheat are the basis of the flexible processing qualities in producing a wide range of food products for a large proportion of the world population. Gluten proteins, also named prolamins, are classically divided into gliadins and glutenins, based on different solubilities in an alcohol/water mixture [1]. The gliadins are generally monomeric proteins, divided into three groups, $\alpha / \beta-, \gamma$ - and $\omega$-gliadins, based on their electrophoretic mobilities at low $\mathrm{pH}$ [2]. Glutenins form polymeric proteins stabilized by interchain disulfide bonds. Based on different molecular weights, glutenins can be classified into two groups, high-molecular-weight glutenin subunits (HMW-GS) and low-molecular-weight glutenin subunits (LMW-GS) [2,3]. LMW-GS are further divided into B-, C- and D-group subunits according to their mobilities in sodium dodecyl sulphate polyacrylamide-gel electrophoresis (SDS-PAGE) [4].

In bread wheat, HMW-GS are encoded by genes at the orthologous Glu-1 loci on the long arms of chromosomes $1 A, 1 B$ and $1 D$ (Glu-A1, Glu-B1 and Glu-D1). Each locus possesses two paralogous genes encoding one $\mathrm{x}$ - and one $\mathrm{y}$-type subunit [5]. LMW-GS genes are located at the Glu-A3, Glu-B3 and Glu-D3 loci on the short arms of group 1 chromosomes. The LMW-GS genes at the Glu-3 loci and the gliadin genes at the Gli-1 loci are tightly linked and form gene clusters covering several centimorgans (cMs) [6-8]. Moreover, unlike the simple composition of HMW-GS, LMW-GS are encoded by a complex multigene family without the information of the exact number of genes $[9,10]$. A large number of genes and abundant allelic variations at Glu3 loci and their tight linkage with gliadin genes make it difficult to elucidate the composition and function of LMW-GS genes in bread wheat [4].

SDS-PAGE is widely used to investigate the abundant seed storage proteins in bread wheat. Based on the mobility of proteins in SDS-PAGE gels, whole seed proteins are divided into four groups, HMW-GS, D-group, Bgroup, and C-group. D-group proteins are mainly composed of $\omega$-gliadin proteins, whereas B-group mostly consists of LMW-GS proteins, and C-group comprise $\alpha$, $\beta$ and $\gamma$-type gliadins and several LMW-GS proteins [4]. Based on the different electrophoretic patterns, LMWGS protein alleles encoded by Glu-3 loci are designated alphabetically (e.g., Glu-A3a) [11]. However, identification of the LMW-GS composition in breeding programs remains a significant challenge because determination of LMW-GS alleles with SDS-PAGE needs much experience. This is why the functions of LMW-GS alleles are not well characterized. Gene-specific markers for Glu$A 3$ and Glu-B3 alleles were developed to identify different LMW-GS alleles. However, molecular markers for Glu-D3 alleles are still not available due to the slight differences among alleles [12-17]. Using BAC library screening and proteomics methods, LMW-GS genes in Norin 61 (Glu-A3d, Glu-B3i and Glu-D3c), Glenlea (Glu-A3g, Glu-B3g and Glu-D3c) and Xiaoyan 54 (Glu$A 3 d$, Glu-B3d and Glu-D3c) were identified and characterized [10,18-20]. These studies greatly improved our understanding of the unique genes encoding different LMW-GS alleles in bread wheat. Recently, based on the conserved and polymorphic structure of LMW-GS genes, we developed a LMW-GS gene marker system and a full-length gene cloning method [21,22]. They were successfully used to identify and characterize more than 16 LMW-GS genes in individual wheat varieties [21]. Both methods are helpful in elucidating the composition of Glu-3 alleles in LMW-GS genes of bread wheat.

The effects of glutenin alleles on dough properties and processing qualities were mostly studied in two types of populations: structured populations (e.g., recombinant inbred lines (RILs) and doubled haploid lines) derived from biparental crosses, and non-structured populations, general collections of varieties and breeding lines. Due to the simple composition and easy identification of allelic variants of HMW-GS, the contributions of HMWGS to dough properties and end-use quality were well investigated and widely used in breeding programs [23]. However, HMW-GS alone could not explain the variation in quality among wheat varieties, as LMW-GS also contributed to dough properties [24-31]. For example, 
LMW-GS alleles made a slightly larger contribution than HMW-GS to dough extensibility [32,33]. Compared with HMW-GS, LMW-GS formed highly polymorphic protein complex and contain abundant allelic variation. Using SDS-PAGE and allele-specific primers, LMW-GS alleles were identified in wheat collections or structured populations, and their effects on processing quality were analyzed and discussed. However, controversies were common in regard to different kinds of populations or collections. For example, Cane et al. [34] and Eagles et al. [33] reported that Glu-A3e was correlated with inferior dough resistance and extensibility, whereas Zheng et al. [35] showed that Glu-A3e was a favorable allele for dough-mixing properties. Due to the complex composition of LMW-GS alleles and difficulties in distinguishing LMW-GS from gliadins in SDS-PAGE gels, the molecular genetic mechanisms behind the functional differences of LMW-GS alleles are not well investigated.

In the present study, a set of near-isogenic lines (NILs) containing five Glu-A3 alleles, eight Glu-B3 alleles and five Glu-D3 alleles, was used to study the effects of LMW-GS on the composition of glutenin macropolymers (GMP), dough properties, and pan bread making quality. These NILs were investigated using SDS-PAGE, the LMW-GS gene marker system and twodimensional gel electrophoresis (2D-PAGE) for identifying the composition of LMW-GS genes and proteins in each LMW-GS allele, and analyzing their association with dough properties and bread-making quality.

\section{Results}

\section{Separation of LMW-GS proteins in Aroona NILs using SDS-PAGE}

The glutenin alleles in the flour of 16 Aroona NILs were separated by SDS-PAGE (Figure 1a). Aroona possessed five HMW-GS proteins, viz., $1,7+9$ and $2+12$, encoded by genes at Glu-A1, Glu-B1 and Glu-D1 loci, respectively. All NILs had the same HMW-GS as Aroona, and their unique LMW-GS and gliadin bands were labeled in Figure 1a. Four Glu-A3 NILs possessed unique LMWGS bands at the B-group region from Aroona (Glu-A3c). Aroona-Glu-A3d and Aroona-Glu-A3e also contained specific gliadin bands (Figure 1a). Among eight Glu-B3 NILs, Aroona (Glu-B3b), Aroona-Glu-B3a, B3f and B3g shared similar B-group proteins, whereas Aroona-Glu$B 3 c, B 3 d, B 3 h$ and B3i showed another group of electrophoretic patterns (Figure 1a). Among the latter four NILs, Aroona-Glu-B3c possessed the lowest quantity of B-group LMW-GS proteins (Additional file 1: Figure S1), especially the protein with the largest molecular weight. Four Glu-D3 NILs and Aroona shared the same B-group LMW-GS proteins (Figure 1a). Aroona-Glu-D3b and D3d had the same protein bands and contained one unique LMW-GS from Aroona. Except for one gliadin protein, the electrophoretic pattern of Aroona-Glu-D3a was the same as those of AroonaGlu-D3b and D3d. Aroona-Glu-D3f produced a quite different protein pattern in the C-group region compared with other Glu-D3 NILs. Generally, the Glu-3 loci were tightly linked with Gli-1 loci [6-8], and it was difficult to break this linkage through genetic recombination in conventional crosses. Thus, each Glu-3 NIL not only possessed unique LMW-GS but also contained 1 or 2 specific gliadins.

\section{Dissecting genes encoding LMW-GS alleles in the Aroona NILs}

To analyze the genes encoding LMW-GS alleles in Aroona NILs, we investigated the 16 Aroona NILs using a previously developed LMW-GS gene marker system, that was efficient in separating LMW-GS genes in bread wheat (Figure 2; Table 1) [21,22]. Eighteen LMW-GS genes were identified in Aroona, including four Glu-A3 genes (A3-391, A3-400, A3-502-2 and A3-620), four Glu$B 3$ genes (B3-530-2, B3-578, B3-607 and B3-621-1), and eight Glu-D3 genes (D3-385, D3-393, D3-394, D3-432,
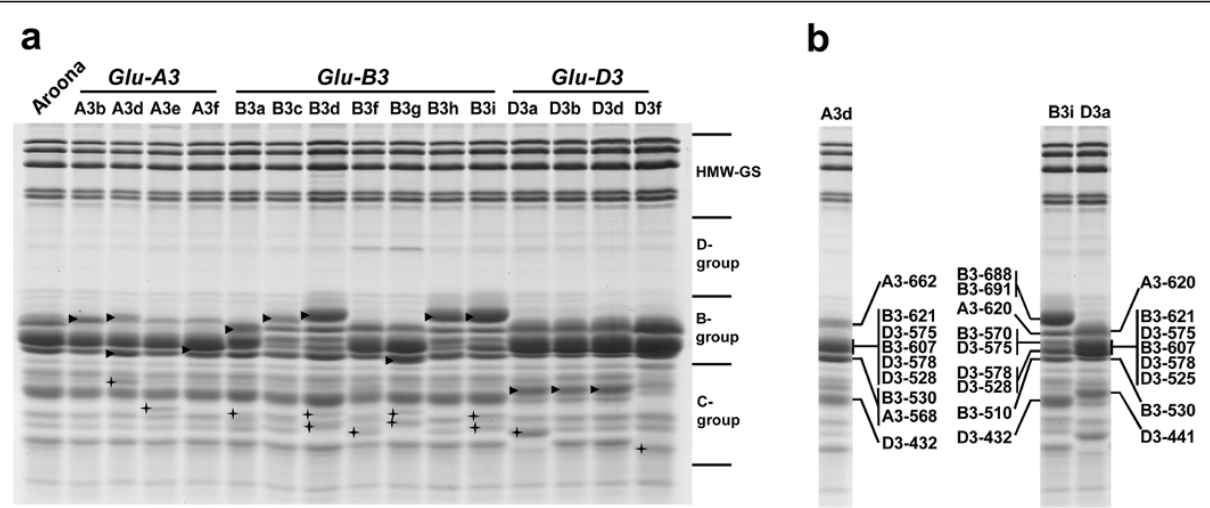

Figure 1 a) Separation of glutenin proteins from flour of Aroona NILs. Arrowheads mark unique LMW-GS protein bands in individual NILS, and asterisks label specific gliadin bands. b) Identification of LMW-GS bands in Aroona-Glu-A3d, Aroona-Glu-B3i and Aroona-Glu-D3a. 


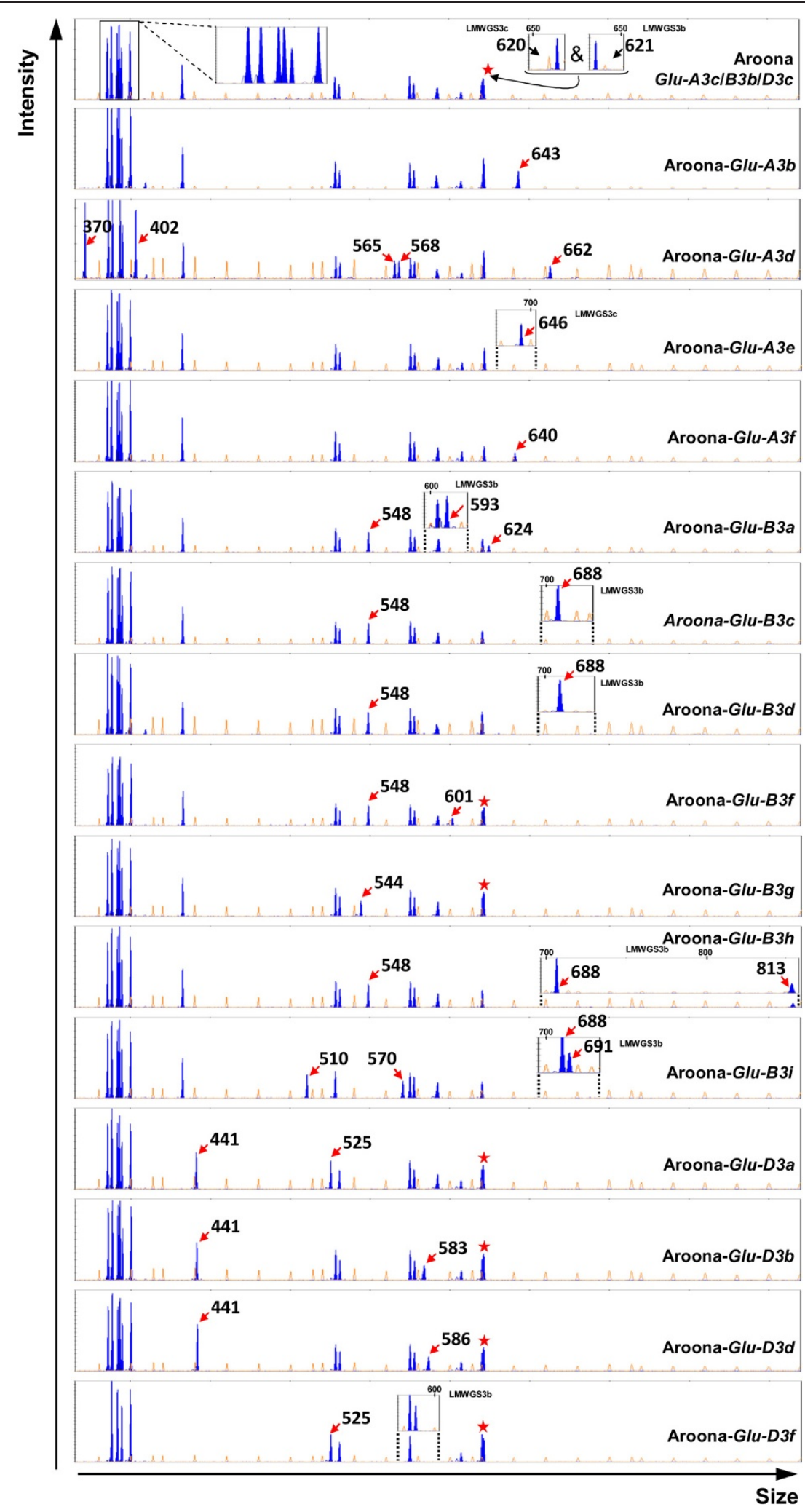

Figure 2 Separation of LMW-GS genes in Aroona NILs using the LMW-GS gene molecular marker system. The marker system contained three sets of primers, LMWGS1, LMW-GS2 and LMWGS3. The main data were derived from primer set LMWGS1, and the added figures were from the primer LMWGS3b or LMWGS3c. The peaks marked with the asterisk comprise two genes, A3-620 and B3-621. The arrows indicate unique genes in each Aroona NIL. 
D3-528, D3-575, D3-578-1 and D3-591) (Table 1). The other two novel genes corresponding to DNA fragments 388 and 410 present in all 16 lines (Table 1) were pseudogenes with premature termination codons in the CDS regions. Among 16 Aroona NILs, Aroona-Glu-D3f possessed 14 unique genes, with the least number of LMW-GS genes, whereas Aroona-Glu-A3d had the largest number of LMW-GS genes (20 genes). Comparison of the LMW-GS genes among NILs indicated that each NIL differed from Aroona at only one Glu-3 locus. With the help of the complete gene sequences available in Aroona NILs and some other wheat varieties $[10,13,14,17-19,21]$, the composition of LMW-GS genes in each Aroona NIL was well characterized.

For Glu-A3 alleles, 4-6 genes were identified in Aroona (Glu-A3c) and four Glu-A3 NILs (Table 1; Figure 2). Except for Aroona-Glu-A3d, these NILs possessed LMW-GS genes A3-391 and A3-400 (Table 1; Figure 2), which were m-type pseudogenes with premature termination codons. The other genes were i-type genes. The A3-502 gene contained four allelic variants with unique SNPs and InDels, i.e., A3-502-1, A3-502-2, A3-502-3 and A3-502-4. All A3-502 allelic variants and the $A 3-640$ gene were pseudogenes, containing premature termination codons in the coding sequences. The i-type genes, A3-643, A3-620, A3-646 and $A 3-573$, were the only active genes in Aroona-Glu-A3b, $A 3 c, A 3 e$ and $A 3 f$, respectively. Sequence alignments showed that $A 3-620$ and $A 3-643$ shared high identity (> 99\%) differing only in one InDel and one SNP, whereas A3-573 and A3-646 were greatly different from each other and the other i-type genes (Additional file 1: Figure S2). A3-573 was much shorter than the other i-type proteins due to deletion of three repetitive units and several glutamine residues in repetitive regions, and lacking GTFLQPH in the C-terminal domain (Additional file 1: Figure S2). Aroona-Glu-A3d contained six unique LMW-GS genes (Table 1; Figure 2). For two m-type genes, $A 3-370$ was a pseudogene, whereas $A 3-402$ contained an intact coding sequence that could be expressed in the developing grain [18]. The other four genes, A3-484, A3-565, A3-568 and $A 3-662$ were i-type genes, and among them $A 3-568$ and A3-662 were active. Both genes contained unique SNPs in the C-terminal domain and different lengths of repetitive region compared to other i-type genes (Additional file 1: Figure S2). Thus, compared to only one LMW-GS gene expressed in the other Glu-A3 alleles, Glu-A3d contained three active genes, one m-type (A3-402) and two i-type (A3-568 and A3-662) LMW-GS genes. Except for A3-402, the active genes at the Glu-A3 locus were i-type genes. These i-type genes encoded B-group LMW-GS, producing different electrophoretic patterns in the B-group region among Glu-A3 NILs (Figure 1).

For Glu-B3 alleles, 3-5 LMW-GS genes were identified in Aroona (Glu-B3b) and seven Glu-B3 NILs. Gene
B3-530 was present in all Aroona NILs and four allelic variants (B3-530-1, -2, -3 and B3-510) were identified (Table 1). Sequence alignment showed that the deduced protein sequences of the three B3-530 allelic variants differed in only four amino acids, whereas B3-510 contained a deletion in the repetitive domain and differed by seven amino acids from the B3-530 proteins (Additional file 1: Figure S3). B3-544, B3-593, B3-601 and B3-607 were allelic variants, sharing over $99 \%$ of identity and their deduced protein sequences differed only in the repetitive domain with the deletion of glutamine residues or one repetitive unit (Additional file 1: Figure S4). B3-621-1, B3621-2 and B3-624 genes were highly conserved and their proteins differed only in one InDel of a glutamine residue in the repetitive domain and one amino acid at the $C$ terminal region (Additional file 1: Figure S5). The B3-688 gene was quite distinct, differing from B3-621 and B3-624 in several SNPs and InDels (Additional file 1: Figure S5). Moreover, B3-688 varied among Aroona-Glu-B3c, B3d, $B 3 h$ and $B 3 i$, and the protein products differed in seven amino acids and two InDels (Additional file 1: Figure S5). Among Glu-B3 genes, B3-548, B3-578 and B3-813 were pseudogenes with premature termination codons, whereas the other genes contained intact ORFs (Open Reading Frame). Sequence analysis showed that B3-530, B3-510, $B 3-548$ and B3-570 were m-type genes and the others were s-type genes. Based on the differences in s-type genes, Glu-B3 NILs were classified into two groups. Aroona-Glu-B3a, B3b, B3f and B3g contained B3-578, B3$544 / 593 / 601 / 607$, and $B 3-621 / 624$, and the others had longer sequences of genes, B3-688 and B3-691/813/Null (Table 1). This classification was consistent with the different electrophoretic patterns contrasting the two groups (Figure 1a).

Aroona-Glu-B3a, B3b, B3f and B3g had similar LMW-GS compositions, but each of them possessed unique allelic variants (Table 1; Figure 2). Both Aroona-Glu-B3f and B3g contained B3-530-3, but they differed in B3-544, B3-601, B3621-1 and B3-621-2 genes (Table 1; Additional file 1: Figures S4 and S5).Aroona-Glu-B3f and Aroona (Glu-B3b) shared the same B3-621-1, but possessed different genes B3-601/ B3-530-3 and B3-607/B3-530-2, respectively (Table 1; Additional file 1: Figures S3 and S4). On the other hand, Aroona-Glu-B3c, B3d, B3h, and B3i also differed in active genes and their allelic variants. Aroona-Glu-B3c and $B 3 d$ contained B3-530-1, but their B3-688 proteins differed in five amino acids (Table 1; Additional file 1: Figure S5). Aroona-Glu-B3i was unique, containing four active LMWGS genes B3-510, B3-570, B3-688-4 and B3-691 (Table 1; Figure 2). B3-570 was only present in Glu-B3i, encoding an m-type LMW-GS with the N-terminal domain METSQIPGLEKPS. Although B3-688-4 and B3-691 were different genes, they shared about $99 \%$ identity and B3-691 is formally reported for the first time. 
Table 1 LMW-GS genes identified in 16 Aroona NILs using the LMW-GS gene molecular marker system

\begin{tabular}{|c|c|c|c|c|c|c|c|c|c|c|c|c|c|c|c|c|c|c|c|c|c|c|c|}
\hline \multirow{2}{*}{$\frac{\text { Line }}{\text { Aroona(Glu-A3c, B3b, D3c) }}$} & \multicolumn{6}{|c|}{ Genes at Glu-A3 locus } & \multicolumn{7}{|c|}{ Genes at Glu-B3 locus } & \multicolumn{8}{|c|}{ Genes at Glu-D3 locus } & \multicolumn{2}{|c|}{ New genes } \\
\hline & 391 & 400 & $502-2$ & & & $620^{a}$ & $530-2$ & & & 578 & 607 & $621-1$ & & 385 & 393 & 394 & 432 & 528 & 575 & $578-1$ & 591 & 388 & 410 \\
\hline Aroona-Glu-A3b & 391 & 400 & $502-1$ & & & 643 & $530-2$ & & & 578 & 607 & $621-1$ & & 385 & 393 & 394 & 432 & 528 & 575 & $578-1$ & 591 & 388 & 410 \\
\hline Aroona-Glu-A3d & 370 & 402 & 484 & 565 & 568 & 662 & $530-2$ & & & 578 & 607 & $621-1$ & & 385 & 393 & 394 & 432 & 528 & 575 & $578-1$ & 591 & 388 & 410 \\
\hline Aroona-Glu-A3e & 391 & 400 & $502-3$ & & & 646 & $530-2$ & & & 578 & 607 & $621-1$ & & 385 & 393 & 394 & 432 & 528 & 575 & $578-1$ & 591 & 388 & 410 \\
\hline Aroona-Glu-A3f & 391 & 400 & $502-4$ & & 573 & 640 & $530-2$ & & & 578 & 607 & $621-1$ & & 385 & 393 & 394 & 432 & 528 & 575 & $578-1$ & 591 & 388 & 410 \\
\hline Aroona-Glu-B3a & 391 & 400 & $502-2$ & & & 620 & $530-1$ & 548 & & 578 & 593 & 624 & & 385 & 393 & 394 & 432 & 528 & 575 & $578-1$ & 591 & 388 & 410 \\
\hline Aroona-Glu-B3C & 391 & 400 & $502-2$ & & & 620 & $530-1$ & 548 & & & & $688-1$ & & 385 & 393 & 394 & 432 & 528 & 575 & $578-1$ & 591 & 388 & 410 \\
\hline Aroona-Glu-B3d & 391 & 400 & $502-2$ & & & 620 & $530-1$ & 548 & & & & $688-2$ & & 385 & 393 & 394 & 432 & 528 & 575 & $578-1$ & 591 & 388 & 410 \\
\hline Aroona-Glu-B3f & 391 & 400 & $502-2$ & & & 620 & $530-3$ & 548 & & 578 & 601 & $621-1$ & & 385 & 393 & 394 & 432 & 528 & 575 & $578-1$ & 591 & 388 & 410 \\
\hline Aroona-Glu-B3g & 391 & 400 & $502-2$ & & & 620 & $530-3$ & & & 578 & 544 & $621-2$ & & 385 & 393 & 394 & 432 & 528 & 575 & $578-1$ & 591 & 388 & 410 \\
\hline Aroona-Glu-B3h & 391 & 400 & $502-2$ & & & 620 & $530-2$ & 548 & & & & $688-3$ & 813 & 385 & 393 & 394 & 432 & 528 & 575 & $578-1$ & 591 & 388 & 410 \\
\hline Aroona-Glu-B3i & 391 & 400 & $502-2$ & & & 620 & 510 & & 570 & & & $688-4$ & 691 & 385 & 393 & 394 & 432 & 528 & 575 & $578-1$ & 591 & 388 & 410 \\
\hline Aroona-Glu-D3a & 391 & 400 & $502-2$ & & & 620 & $530-2$ & & & 578 & 607 & $621-1$ & & 385 & 393 & 394 & 441 & 525 & 575 & $578-2$ & 591 & 388 & 410 \\
\hline Aroona-Glu-D3b & 391 & 400 & $502-2$ & & & 620 & $530-2$ & & & 578 & 607 & $621-1$ & & 385 & 393 & 394 & 441 & 528 & 575 & $578-2$ & 583 & 388 & 410 \\
\hline Aroona-Glu-D3d & 391 & 400 & $502-2$ & & & 620 & $530-2$ & & & 578 & 607 & $621-1$ & & 385 & 393 & 394 & 441 & 528 & 575 & $578-2$ & 586 & 388 & 410 \\
\hline Aroona-Glu-D3f & 391 & 400 & $502-2$ & & & 620 & $530-2$ & & & 578 & 607 & $621-1$ & & - & - & 394 & - & 525 & 575 & 578 & - & 388 & 410 \\
\hline
\end{tabular}

${ }^{a}$ Bold numbers indicate active LMW-GS genes. 
At the Glu-D3 locus, eight genes were identified from individual NILs except Aroona-Glu-D3f (Table 1). D3-393 and D3-583/586/591 had premature termination codons. All the other genes were widely reported and were expressed in developing grains $[10,17,18,20]$. D3-385, D3-394 and D3-575 were highly conversed among the Aroona NILs. D3-432 and D3-441 were allelic variants with over $99 \%$ identity, and differed in only two SNPs and two InDels. Allelic variants D3-525 and D3-528 were identical, except for one InDel. Two allelic variants, D3-578-1 and D3-578-2 differed by eight SNPs (Table 1). Compared with the active Glu-D3 genes in Aroona (Glu-D3c), D3-441 and D3-578-2 were present in Aroona-Glu-D3b and D3d, which possessed the same active LMW-GS genes (Table 1; Figure 2). Besides D3-441 and D3-578-2, Aroona-Glu-D3a contained unique gene D3-525 in contrast to Aroona. In Aroona-Glu-D3f, only four LMW-GS genes were identified; the other four genes might be absent due to deletion, substitution or other mutations (Table 1; Figure 2).

Each Aroona NIL possessed unique LMW-GS alleles. The gene compositions of these LMW-GS alleles were dissected, all the LMW-GS gene sequences were characterized, and the proteins sequences were deduced (Table 1; Figure 2). Based on the size of the deduced LMW-GS and the LMWGS 2D-PAGE spots identified from Xiaoyan 54, Jing 411 and Norin $61[18,19]$, LMW-GS genes were assigned to protein bands in SDS-PAGE gels (Figure 1b). The active i-type genes at the Glu-A3 locus, all the active genes at Glu-B3 and the genes D3-525/528, D3-575 and D3-578 at Glu-D3 encoded B-group LMW-GS, whereas the protein products of the other active genes belonged to the C-group. Moreover, B3-621/624, B3-593/601/607, D3-575 and D3-578 encoded proteins with similar molecular weights (38$40 \mathrm{kDa}$ ), and ran synchronously on SDS-PAGE, forming the thickest protein bands in Aroona-Glu-B3a, B3b and B3f. $B 3-688$ genes encoding the longest protein conferred the unique protein pattern on Aroona-Glu-B3d, B3h and B3i. (Figure 1).

\section{Comparison of whole proteins among LMW-GS NILs using 2D-PAGE}

The data from SDS-PAGE and the LMW-GS marker system showed that each Glu-3 allele contained several unique LMW-GS and gliadin genes. To analyze the composition of LMW-GS and gliadin proteins in individual NILs, we performed 2D-PAGE analysis of the whole flour proteins (Figures 3, 4 and 5). In the previous studies, using 2D-PAGE coupled with MS or N-terminal sequencing technology, LMW-GS proteins in Xiaoyan 54, Jing 411, Norin 61 and Butte 86 were successfully identified $[18,19,36]$. These data greatly contributed to the identification of flour proteins in the Aroona NILs.
Among five Glu-A3 NILs, Aroona-Glu-A3b and Aroona $(G l u-A 3 c)$ shared the same protein patterns, except for one gliadin spot, which was in agreement with the similar composition of LMW-GS genes and the high identity between A3-620 and A3-643 (Figure 3a). Compared with Aroona, Aroona-Glu-A3d contained three unique LMWGS, A3-402, A3-568 and A3-662, and two gliadin spots were absent (Figure 3a). For Aroona-Glu-A3e, the protein product of the unique i-type gene $A 3-646$ was too little to be detected, but three unique gliadin spots were present in 2D-PAGE (Figure $3 \mathrm{~b}$ ). Aroona-Glu-A3f also contained one unique i-type protein (A3-573) and one gliadin spot different from Aroona (Figure $3 \mathrm{~b}$ ). Thus, accompanying the unique LMW-GSs in individual Glu-A3 NILs, generally 1-2 gliadin spots were different among them. The only exception was Aroona-Glu-A3e which possessed the highest number of gliadins and the lowest quantity (none) of i-type LMW-GS among the Glu-A3 NILs (Figure $3 \mathrm{~b}$ ).

Among eight Glu-B3 NILs, Aroona-Glu-B3c, B3d, B3h and $B 3 i$ shared similar protein spot patterns on 2D-PAGE, consistent with their similar electrophoretic patterns on SDS-PAGE (Figures 1a and 4). Compared with the protein spot pattern of Aroona (Glu-B3b), Aroona-Glu-B3c, B3d, $B 3 h$ and $B 3 i$ contain the unique B3-688 proteins (Figure 4). The B3-688 protein spot in Aroona-Glu-B3c was much weaker than those in the other three NILs (Additional file 1: Figure S6). Except for LMW-GS proteins, only one or two small gliadin spot differences were identified among the four NILs (Figure 4). The other three Aroona NILs (i.e., Aroona-Glu-B3a, B3f and B3g) had similar protein spot patterns with Aroona (Glu-B3b; Figure 4). Among these four NILs, a unique LMW-GS protein B3-544 was detected in Aroona-Glu-B3g, whereas the other three alleles shared the same LMW-GS protein spot patterns (Figure 4). Thus, the 2D-PAGE patterns of Aroona and seven Glu-B3 NILs suggested that their different proteins mainly were unique LMW-GS proteins rather than gliadins.

For the Glu-D3 NILs, the protein spots encoded by the D3-441 gene in Aroona-Glu-D3a,D3b and D3d had larger molecular weights and pIs than its allelic variant D3-432 in Aroona (Figure 5). No other different LMW-GS spots were detected among these Glu-D3 NILs, and Aroona-Glu-D3a and D3d, each contained only one unique gliadin spot (Figure 5). The AroonaGlu-D3f allele was unique, lacking two LMW-GS proteins (i.e., D3-385 and D3-441) and at least three medium gliadin spots (Figure 5). The absence of four LMW-GS genes was also observed using the LMW-GS gene marker system (Table 1). Thus, except for Aroona-Glu-D3f, only one or two different protein spots were detected among Glu-D3 NILs, and the proteins encoded by genes at the Glu-D3 locus were highly conserved. 

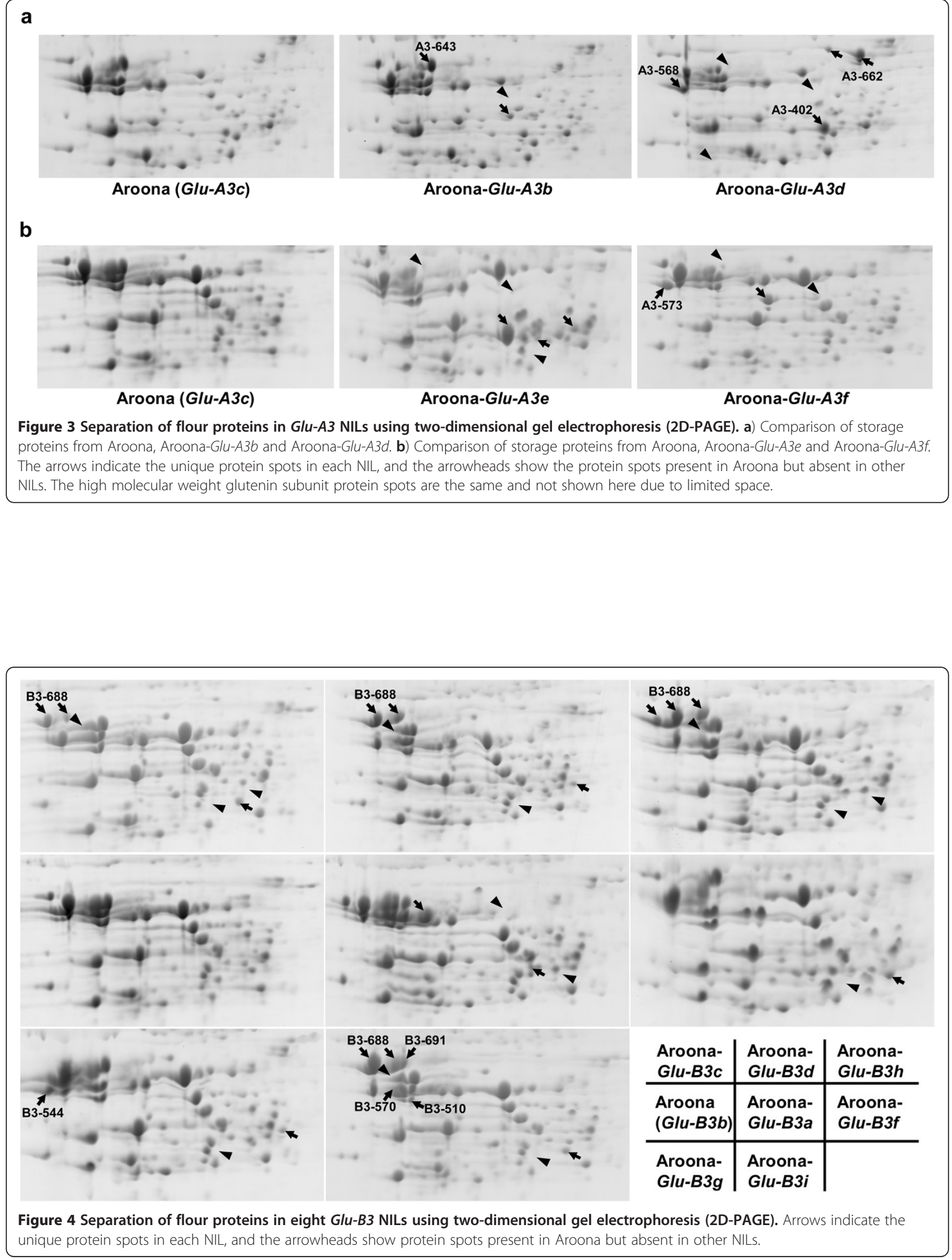


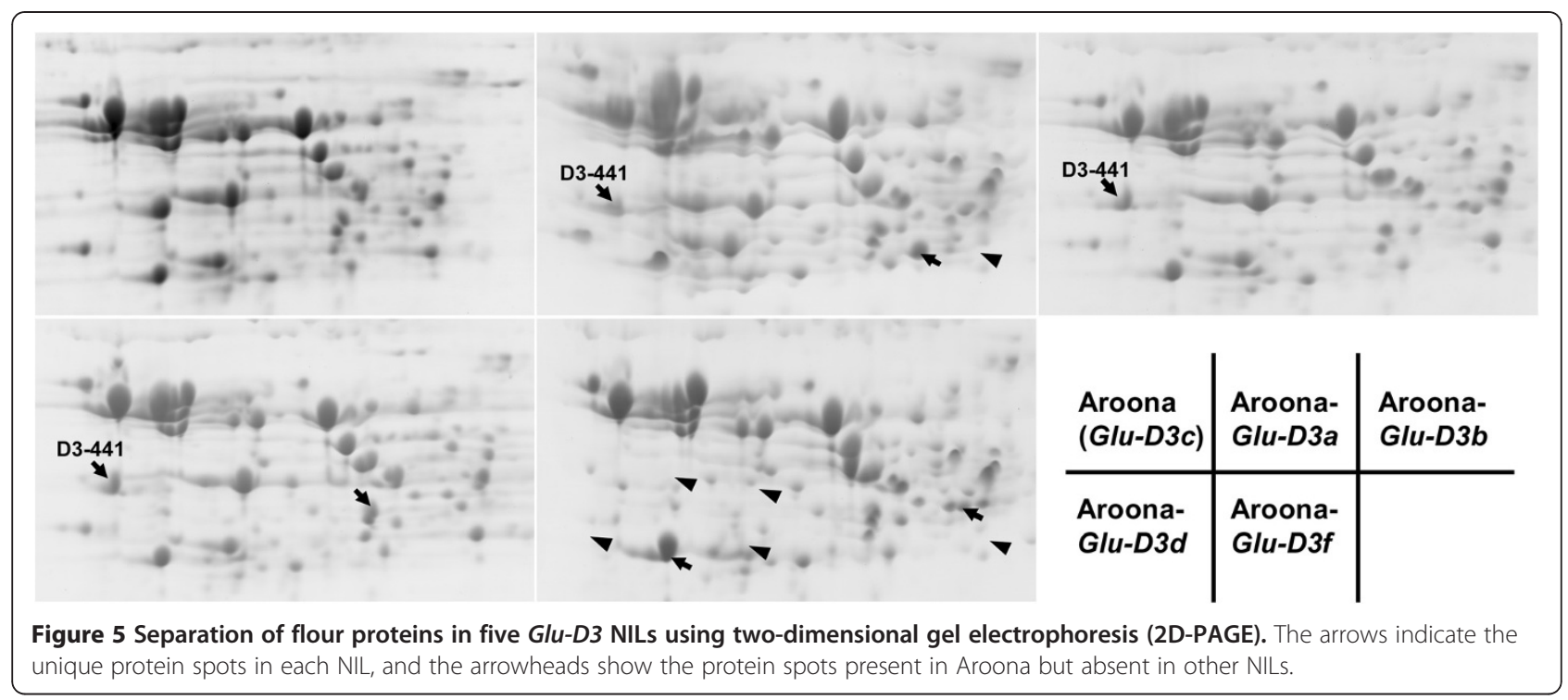

\section{Quality properties of the LMW-GS NILs}

Dough properties such as Zeleny-sedimentation value (ZSV), Farinograph and Extensograph parameters, GMP parameters, and pan bread quality parameters, were measured on the Aroona NILs (Table 2). The 16 genotypes showed significant differences $(P<0.05$ or $P<0.01)$ in most quality parameters, suggesting that genotype had an important influence on variation in wheat quality properties (Additional file 2: Table S1). Significant differences in some parameters (e.g., kernel protein, ZSV, Farinograph water absorption, glutenin and gliadin contents, and external bread color and structure) were also observed between the two locations (Additional file 2: Table S1). However, no significant interaction effects between genotypes and locations were detected for most quality parameters, except for external color of bread and Farinograph development time (Additional file 2: Table S1). Analysis of variance (ANOVA) and multiple comparisons of all quality parameters were performed among all NILs within each Glu-3 group (Table 2, Additional file 2: Tables S2, S3 and S4).

ANOVA showed that the Glu-A3 NILs were significantly different in most quality parameters (Table 2). This suggested that Glu-A3 alleles affected the bread-making quality of bread wheat. Subsequent multiple comparisons indicated that Aroona-Glu-A3e always produced the worst quality parameters, e.g., ZSV, Farinograph stability time (ST), Extensograph maximum resistance (Rmax), Extensograph extensibility (Ext), percentage of SDS-unextractable fraction in total polymeric protein (\%UPP), and pan bread

Table 2 F values of one way ANOVA of wheat quality parameters within each Glu-3 group ${ }^{\text {a }}$

\begin{tabular}{|c|c|c|c|c|c|c|c|c|c|c|}
\hline Group & LV & LVS & EC & Sha & IC & Smo & Spr & Str & TF & PBTS \\
\hline Aroona-Glu-A3 & $5.31^{*}$ & 2.33 & 0.7 & 3.35 & 1.14 & $12.14^{* *}$ & 0.67 & $6.45^{*}$ & 2 & $4.75^{*}$ \\
\hline Aroona-Glu-B3 & $2.85^{*}$ & 2.07 & 0.8 & 1.57 & 1.28 & 2.09 & 0.75 & $5.09^{* *}$ & 2 & $5.78^{* *}$ \\
\hline Aroona-Glu-D3 & 3.74 & 2.29 & 1.71 & 2.57 & 1 & 1 & 0.33 & 0.52 & 0.12 & 0.68 \\
\hline Group & KP & ZSV & ST & DT & Wab & Rmax & $\mathrm{EA}$ & Ext & & \\
\hline Aroona-Glu-A3 & 3.3 & $59.35^{* *}$ & $6.55^{*}$ & $11.57^{* *}$ & $27.98^{* *}$ & $10.34^{* *}$ & $13.46^{* *}$ & $4.51^{*}$ & & \\
\hline Aroona-Glu-B3 & 2.54 & $43.89^{* *}$ & $6.19^{* *}$ & $8.47^{* *}$ & $5.4^{* *}$ & $9.97 * *$ & $10.06^{* *}$ & 2.34 & & \\
\hline Aroona-Glu-D3 & 3.01 & 0.85 & 2.47 & 3.05 & $4.04^{*}$ & 2.58 & 1.18 & 2.33 & & \\
\hline Group & Glutenin & Gliadin & Gli/Glu & EPP & UPP & \%UPP & & & & \\
\hline Aroona-Glu-A3 & 0.28 & 3.13 & $4.62^{*}$ & 2.87 & $7.1^{* *}$ & $9.58^{* *}$ & & & & \\
\hline Aroona-Glu-B3 & 0.55 & $8.81^{* *}$ & $4.65^{* *}$ & 2.14 & $3.15^{*}$ & $3.63^{*}$ & & & & \\
\hline Aroona-Glu-D3 & 3.64 & 0.32 & $6.55^{*}$ & 1.51 & 1.53 & 1.48 & & & & \\
\hline
\end{tabular}

* Significant at $P<0.05$; * Significant at $P<0.01$.

a LV: Loaf volume $\left(\mathrm{cm}^{3}\right)$, LVS: Loaf volume score, EC: External color, Sha: Shape, IC: Inner color, Smo: Smoothness, Spr: Springiness, Str: Structure, TF: Taste flavor, PBTS: Pan bread total score, KP: Kernel protein (\%, 14\% m.b.), ZSV: Zeleny sedimentation value (mL), ST: Farinograph stability time (min), DT: Farinograph development time ( $\mathrm{min})$, Wab: Farinograph water absorption (\%), Rmax: Extensograph maximum resistance (B.U.), EA: Energy area (cm²), Ext: Extensograph extensibility (mm), EPP: Extractable glutenin polymeric protein; UPP: Unextractable glutenin polymeric protein; Gli/Glu: Ratio of gliadin to glutenin; \%UPP = UPP/ $(\mathrm{UPP}+\mathrm{EPP}) \times 100$. 
total score (PBTS), whereas the other five Glu-A3 NILs had similar values for Rmax, ST, \%UPP and Ext (Figure 6). Although Aroona-Glu-A3d had the largest ZSV, it showed only moderate PBTS (Figure 6).

Glu-B3 NILs showed large differences in the most important quality parameters (Table 2), indicating that Glu-B3 alleles were also significantly associated with bread-making quality. Among eight Glu-B3 NILs, Aroona-Glu-B3g produced the highest PBTS, ZSV, ST, Rmax, \%UPP and some other parameters (Figure 6, Additional file 2: Tables S2, S3 and S4). Similarly, Aroona (Glu-B3b) and Aroona-Glu-B3i had higher quality parameters than the other Glu-B3 NILs, excluding Aroona-Glu-B3g. In contrast, Aroona-Glu-B3c performed the lowest in ZSV, ST, Rmax, \%UPP, PBTS, and some other parameters (Figure 6; Additional file 2: Tables S2, S3 and S4), suggesting that Glu-B3c was an undesirable allele for bread-making quality. The other Glu-B3 NILs (Aroona-Glu-B3a, B3d, B3f and B3h) possessed similar moderate quality parameters, without significant differences.

Five Glu-D3 NILs produced similar quality parameters, without significant differences, except for Farinograph water absorption and the ratio of gliadin to glutenin (Table 2). This was consistent with the similar composition of LMW-GS genes and proteins among Glu-D3 NILs.

\section{Discussion}

In the present study, SDS-PAGE, 2D-PAGE and LMWGS gene molecular marker system were used for characterization of LMW-GS genes and proteins in 16 Aroona NILs. Each NIL contained a unique LMW-GS allele. The genetic and protein compositions of each LMW-GS allele were dissected. In addition, we analyzed the functional differences among the LMW-GS alleles in bread-making quality using these NILs. The molecular mechanisms behind the functional differences were discussed based on the characterization of LMW-GS genes and proteins in the NILs.

\section{Characterization of LMW-GS genes and proteins in the NILs}

SDS-PAGE is a useful method to accurately identify HMW-GS in bread wheat, but is inefficient for separating LMW-GS alleles, because of the presence of large numbers of LMW-GS proteins with similar mobilities with each other and with gliadins (Figure 1b). In the present study, the LMW-GS gene marker system was successfully used to separate 14-20 LMW-GS from each NIL, and also distinguished 16 Glu-3 NILs, except for Aroona-Glu-B3c and B3d without the length polymorphisms among B3-688-1 and B3-688-2 (Table 1; Figure 2). These results indicated that the LMW-GS gene marker system was efficient and accurate not only in separating members of the LMW-GS gene family [21], but also in distinguishing allelic variants of individual LMW-GS genes. Recently, allele-specific markers were widely used in distinguishing Glu-A3 and Glu-B3 alleles [12-14]. However, the high conservation among Glu-D3 allelic variants made it difficult to develop allele-specific markers for discriminating different allelic variants. Because these allelic variants showed DNA sequence polymorphisms in length, the LMW-GS gene marker system worked well in dissecting the complex genes and allelic variants at the Glu-D3 locus (Table 1). On the other hand, compared with only one gene in individual alleles identified with gene-specific primers $[13,14]$, almost all the genes in Glu-3 alleles were displayed using the LMW-GS gene marker system (Table 1). Identification of all genes in each allele will greatly contribute to an understanding of the molecular mechanism determining functional differences among Glu-3 alleles in bread wheat.

In the present study, Aroona and its NILs contained most of the Glu-3 allelic variations identified from the worldwide wheat germplasm in a cooperative program [12]. Each LMW-GS allele was encoded by several linked genes or haplotypes $[13,14,16,17,21,22]$. The dissection of LMW-GS genes in Aroona NILs would facilitate the haplotype analysis of LMW-GS genes in common wheat. At the Glu-A3 locus, besides two m-type genes, 2-4 itype genes were identified from individual Glu-A3 alleles. Unlike the conserved m-type genes, i-type genes were completely different among five alleles. These i-type genes might be tightly linked, forming unique haplotype in each Glu-A3 allele, e.g. A3-484/A3-565/A3$568 / A 3-662$ in Glu-A3d (Table 1) $[13,18,19,21]$. At the Glu-B3 locus, 1-2 m-type gene(s) and 1-3 s-type gene (s) were characterized in individual alleles. Generally, these s-type genes could be divided into two groups, one containing B3-578/B3-544/593/601/607/B3-621/624, the other having B3-688/B3-691/813/N (Table 1, Figure 2). These s-type genes in each group might cosegregate and form unique haplotypes in bread wheat [14]. Among four allelic variants of m-type gene B3-530, B3-530-2 was present in both Aroona $(G l u-B 3 b)$ and AroonaGlu-B3h, containing difference s-type haplotypes B3578/B3-607/B3-621-1 and B3-688/B3-813, respectively. Thus, at the Glu-B3 locus, the $\mathrm{m}$ - and s-type genes appeared not to be tightly linked (Table 1; Figure 2) $[6,18]$. All the Glu-D3 alleles except Glu-D3f contained eight LMW-GS genes (Table 1). Only one or two allelic variants (D3-432/441, D3-525/528 and D3-5781/-2 were identified for each LMW-GS gene and only a few SNPs were detected among allelic variants. Thus, Glu-D3 genes were highly conserved among wheat varieties [16,17], wherease Glu-A3 and Glu-B3 genes showed high diversities, which were derived 

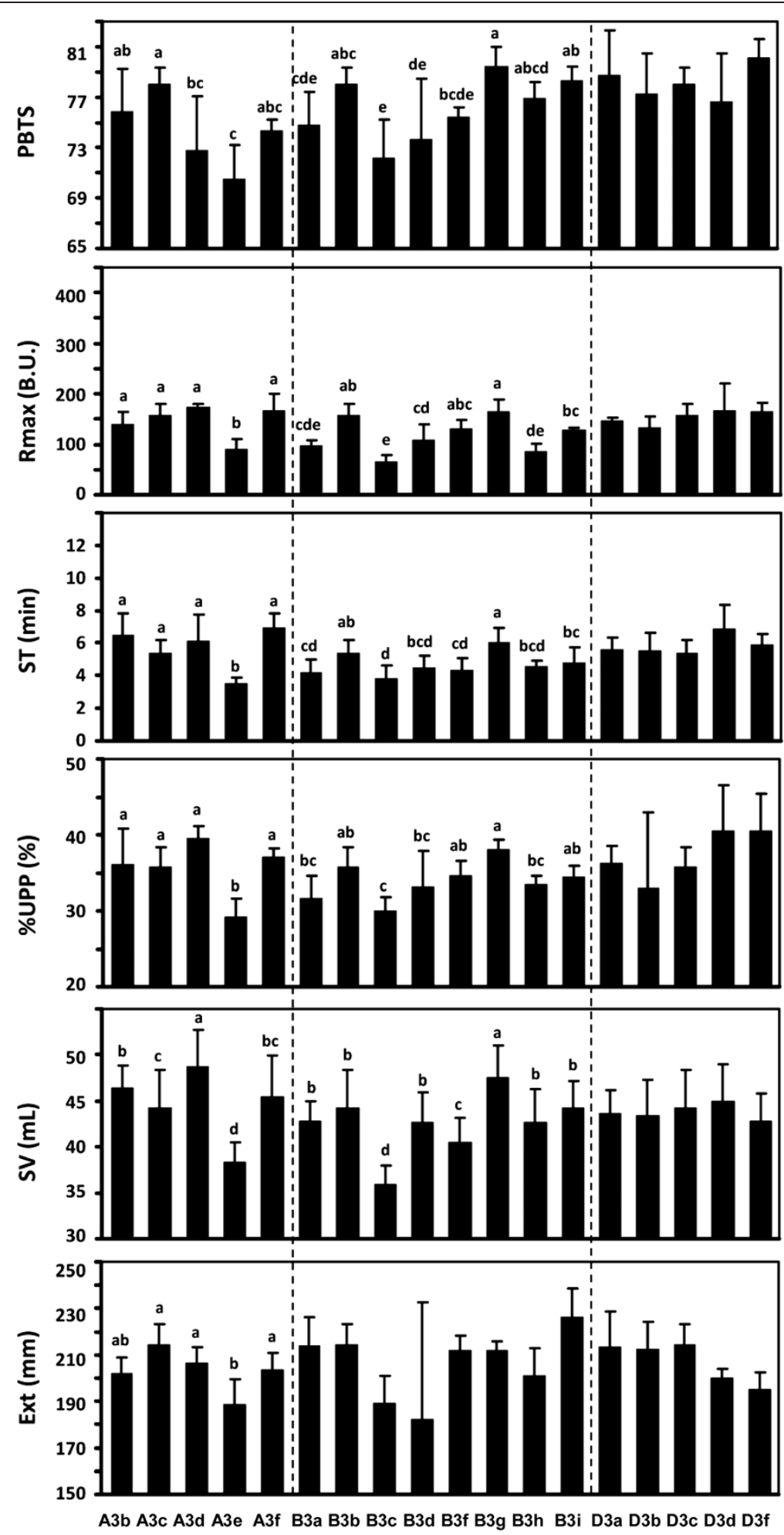

Glu-3 allele

Figure 6 Comparison of wheat quality properties among Glu-A3 NILs, Glu-B3 NILs and Glu-D3 NILs. Statistical analysis of the quality parameters of Aroona NILs for each glutenin locus by ANOVA and Duncan LSR $(P<0.05)$. The bars are labeled by different letters or letter combinations based on multiple statistical comparisons. No statistical significance exists between alleles labeled by one or more identical letters. PBTS, Pan bread total score; Rmax, Extensograph maximum resistance; ST, Farinograph stability time; \%UPP, Percent of SDS-unextractable fraction in total polymeric protein; ZSV, Zeleny-sedimentation value; Ext, Extensograph extensibility. 
from multiple allelic variations of i- and s-type genes, respectively.

To analyze differences in seed storage proteins among LMW-GS NILs, flour proteins were displayed using 2DPAGE (Figures 3, 4 and 5). LMW-GS proteins shared some similar characteristics, such as mostly located in the $\mathrm{B}$-group region and larger pIs than gliadins. Although it is difficult to detect small variation in molecular weight and quantity of each protein spot in 2DPAGE, the composition and allelic variations of seed storage proteins for each Glu-3 allele were successfully characterized. These results were in agreement with the 2D protein patterns of Xiaoyan 54, Jing 411, Norin 61 , Butte 86 and some other varieties identified with LC-MS or N-terminal sequencing $[18,19,36]$. For example, the Glu-A3d and Glu-B3d alleles showed similar electrophoretic patterns to Xiaoyan 54 and Norin 61 in i-type (A3-568 and A3-662) and s-type (B3-688) proteins, respectively $[18,19]$, and $G l u-B 3 g$ shared the same spot pattern of B3-544 with Glenlea [19]. Moreover, comparison of 2D-PAGE patterns showed that, except for the different LMW-GS proteins, only 1-3 unique small gliadin spots were present in individual Aroona NILs (Figures 3, 4 and 5). These results suggested that the differences among the Aroona NILs were mainly derived from allelic variation of LMW-GS genes.

\section{LMW-GS alleles and bread-making quality}

The NIL population was used to study the effects of LMW-GS on dough properties, GMP parameters, and pan bread quality parameters. The results confirmed that the LMW-GS played important roles in determining variation in wheat quality properties. Among the three Glu-3 loci, alleles Glu-A3 and Glu-B3 were of major importance in determining differences in processing qualities among the NILs (Table 2).

\section{Glu-A3 alleles}

Among five Glu-A3 alleles, Glu-A3e (A3-391, A3-400, A3502-3 and A3-646) performed the poorest in almost all quality properties (Table 1, Figure 6; Additional file 2: Tables S2, S3 and S4). This was consistent with previous reports in which Glu-A3e was associated with lower extensibility and Rmax than Glu-A3d, $A 3 b$ and $A 3 c$ [33]. The negative effect of Glu-A3e on dough rheological properties was reported in several studies previously $[24,33,37,38]$. No unique i-type protein band was detected from the Glu-A3e allele using SDS-PAGE (Figure 1a) [39]. The protein product of the $A 3-646$ gene was also not identified in 2D-PAGE although the i-type gene A3-646 in Aroona-Glu-A3e contained the intact ORF (Figure 3b, Additional file 1: Figure S2). Less i-type proteins and more gliadins in
Glu-A3e increased the ratio of gliadin to glutenin and greatly reduced \%UPP (Figure 6; Additional file 2: Table S4), resulting in the worst performance of the Glu-A3e genotype in dough resistance and extensibility and pan bread total score [39]. The other Glu-A3 NILs produced similar quality parameters, including Rmax, ST, Ext and \%UPP. These data indicated that Glu-A3a, A3c, $A 3 d$ and $A 3 f$ all had equivalent positive effects on UPP content and dough resistance and extensibility (Figure 6). Among them, Glu-A3d had a significant effect on high ZSV, which was consistent with results from Xiaoyan 54 and Jing 411 RILs [18]. Some other studies also reported that the Glu-A3d allele had a superior effect on dough strength [40-42]. Compared with only one active gene at the Glu-A3 locus in the other alleles, the Glu-A3d allele possessed three active LMW-GS genes and produced the highest ZSV, Rmax, and \%UPP (Figure 6). The large number of active genes in Glu-A3d might be the basis of the superior performance in wheat quality properties [18].

\section{Glu-B3 alleles}

Our study confirmed the important contribution of the Glu-B3 locus to quality variation of bread wheat varieties. Among eight Glu-B3 alleles, Glu-B3c (B3-530-1, B3-548 and $B 3-688-1)$ produced the lowest quality parameters, including ZSV, ST, Rmax, \%UPP, and PBTS (Figure 6). The inferior effects of $G l u-B 3 c$ allele were also reported in previous studies $[24,33,43,44]$. Glu-B3c and Glu-B3d (B3-530-1, B3-548 and B3-688-2) shared an identical LMW-GS composition, except for five amino acid differences between B3-688-1 and B3-688-2 (Table 1; Figure 2, Additional file 1: Figure S5), but produced significantly different Rmax and ZSV (Figure 6). B3-688-2 appeared to produce better quality properties than $B 3-688-1$. On the other hand, Glu-B3c and Glu-B3h (B3-530-2, B3-548, B3-688-3 and B3-813) also contained similar active LMWGS genes and had only a few SNPs in the B3-530 and B3-688 allelic variants, but Glu-B3h had significantly more positive effects on PBTS and ZSV than Glu-B3c (Figure 6). The 2D-PAGE showed that $G l u-B 3 c$ had a smaller quantity of B3-688-1 protein than Glu-B3d and B3h (Figure 4, Additional file 1: Figure S6). This suggested that the unique SNPs in B3-688-1 might result in lower expression or difficulty in translation and polymerization. The small amount of B3-688-1 might directly cause the higher ratio of gliadin to glutenin and the lower \%UPP for the Glu-B3c allele, finally resulting in its inferior effects on wheat quality (Additional file 2: Tables S2, S3 and S4). Compared to two active genes in the three Glu-B3 alleles above, Glu-B3i contained four active genes, m-type haplotype B3-510/B3-570 and s-type haplotype B3-688/B3-691. Both haplotypes provided the highest number of active genes and lead to the best quality performance among the four alleles [18]. 
The Glu-B3a, B3b, B3f and $B 3 g$ alleles belonged to the same group of s-type haplotypes. They shared similar electrophoretic patterns and each possessed three active LMW-GS genes, but had significantly different effects on quality properties (Figure 6). Glu-B3g (B3-530-3, $B 3-548, B 3-544$ and B3-621-2) produced the best quality parameters, including ZSV, Rmax, ST, \%UPP, LV and PBTS (Figure 6), in agreement with previous reports $[40,42,45]$. Glu-B3b (B3-530-2, B3-548, B3-607 and B3-621-1) also had positive effects on most quality parameters (Figure 6), again consistent with the previous studies [46,47]. The SE-HPLC parameters indicated that the low content of gliadin and the high content of UPP might be the main reasons for the superior performance of Glu-B3b and B3g (Figure 6; Additional file 2: Table S4). And the high content of UPP might be derived from the SNPs or InDels in the nucleotide sequences of three active genes (B3-530-2/3, B3-544/607 and $B 3-621-1 /-2)$, which enhanced the ability of the protein products to form large glutenin macropolymers. More evidence was obtained by comparing Glu-B3f and Glu-B3g. Both alleles contained the same m-type gene, B3-530-3, but had different s-type haplotypes and significant differences in ZSV, ST, UPP, and PBTS. The s-type haplotype B3-578/B3-544/B3-621-2 formed more UPP and produced better bread-making quality than $B 3-578 /$ B3-601/B3-621-1.

\section{Glu-D3 alleles}

The five Glu-D3 alleles produced similar values for almost all quality properties in the present study (Figure 6; Table 2), which confirmed previous reports that Glu-D3 alleles produced similar Rmax and extensibilities among large collections of wheat varieties [24,33,48], although some studies indicated different effects of Glu-D3 alleles on dough strength or mixing properties [27,29,38,49]. Glu-D3a,D3b, D3c and D3d contained similar 2DPAGE spot patterns, and all six active genes were highly conserved among the alleles (> 99\% identities). Their similarity in LMW-GS genes and whole proteins was consistent with their equivalent effects on all quality properties. Although Glu-D3f allele lacks two LMW-GS proteins and three gliadin spots, it produced similar quality properties to the other Glu-D3 alleles (Figure 5; Table 2). These results suggested that D3-385, D3-432 and the three missing gliadins were not related to quality improvement. However, the lack of active genes D3-394 and D3-528 at the Glu-D3 locus in Jing 411 (Glu-D3l) showed significant negative effects on ZSV [18]. Thus, except for Glu-D3l, Glu-D3 alleles appeared to play only minor roles in determining quality variation among bread wheat varieties, and they should be given the lowest priority among LMW-GS alleles in selecting for improved bread-making quality in wheat.

\section{Conclusion}

In the present study, we dissected the genetic and protein composition of 16 LMW-GS NILs, measured the dough property and bread-making quality properties of individual NILs, and performed functional analyses for each allele. Among five Glu-A3 alleles, Glu-A3e (i-type haplotype $A 3-502-3 / A 3-646)$ was inferior with negative effects on all quality properties. Among eight Glu-B3 alleles, Glu-B3b (m-type gene B3-530-2 and s-type haplotype B3-578/B3-607/B3-621-1), Glu-B3g (m-type gene B3-530-3 and s-type haplotype B3-578/B3-544/B3621-2) and Glu-B3i (m-type haplotype B3-510/B3-570 and s-type haplotype B3-688-4/B3-691) were correlated with superior bread-making quality, whereas Glu-B3c (m-type gene B3-530-1 and s-type haplotype B3-688-1/ $N)$ produced inferior quality properties. Among five Glu-D3 alleles, there were no significant differences in all quality parameters measured in the present study. Moreover, all alleles with superior dough properties and pan bread quality also possessed high contents of UPP and \%UPP. Thus, it is possible that LMW-GS alleles determine dough viscoelasticity by modifying the size distribution of glutenin polymers and aggregative properties of glutenins [50]. These results significantly enhance our understanding of the composition of LMWGS, confirm the strong effects of LMW-GS on not only dough extensibility but dough strength, and provide useful information for quality improvement in bread wheat.

\section{Methods}

\section{Plant materials}

The wheat variety Aroona and 15 near isogenic lines (NILs) were kindly provided by Dr. Marie Appelbee and Prof. Ken Shepherd, SARDI Grain Quality Research Laboratory, South Australia. Each NIL contains a unique LMW-GS allele from a donor variety added to Aroona (Additional file 2: Table S5). They were planted at the Xinjiang Academy of Agri-Reclamation Sciences, Shihezi, and Xinjiang Academy of Agricultural Sciences, Urumqi, Xinjiang province, in randomized complete blocks with two replications during the 2010 cropping season.

\section{Analysis of LMW-GS genes}

Genomic DNA of 16 Aroona NILs was extracted from young leaves of seedlings following Saghai-Maroof et al. [51]. The LMW-GS genes were separated by the LMWGS gene molecular marker system [22]. With the help of the LMW-GS genes available from the Aroona NILs and some other wheat varieties $[10,13,14,17,18]$, LMWGS genes were characterized using Lasergene software (DNAStar; http://www.dnastar.com/), ClustalW2 (http:// www.ebi.ac.uk/Tools/msa/clustalw2/), and MEGA 5 software [52]. 


\section{Isolation and separation of LMW-GS proteins}

Glutenin extraction was performed according to the method described by Singh et al. [11]. These proteins were separated by SDS-PAGE using the method described by Sunbrook and Russell [53]. Whole seed proteins were isolated from wheat flour based on the SDS/Phenol method [54] with some modifications. Briefly, proteins in $0.12 \mathrm{~g}$ flour were precipitated with $10 \% \mathrm{TCA}$ /acetone at $-20^{\circ} \mathrm{C}$ overnight. After centrifuging at $20,000 \mathrm{~g}$ at $4^{\circ} \mathrm{C}$ for $15 \mathrm{~min}$, the pellet was washed three times with $80 \%$ acetone, then dried at $50^{\circ} \mathrm{C}$. Whole proteins were extracted with SDS/ Phenol buffer (50\% Tris-phenol pH 8.0, 30\% sucrose, $2 \%$ SDS, $0.1 \mathrm{M}$ Tris- $\mathrm{HCl} \mathrm{pH} 8.0$ and 2\% DTT). The upper phenol phase was transferred into a new $2 \mathrm{~mL}$ tube. A fivefold volume of methanol containing $0.1 \mathrm{M}$ ammonium acetate was added to the tube. The proteins were deposited at $-20^{\circ} \mathrm{C}$ for $10 \mathrm{~min}$ or overnight. After centrifuging at $20,000 \mathrm{~g}$ for $5 \mathrm{~min}$ at $4^{\circ} \mathrm{C}$, the pellet was washed once with $100 \%$ methanol and twice with $80 \%$ acetone before briefly drying in air. The proteins were dissolved in isoelectric focusing (IEF) sample extraction solution and used in 2DPAGE analysis according to Dong et al. [18]. The images of SDS-PAGE were analyzed using NIH ImageJ software program (http://rsb.info.nih.gov/ij/).

\section{Quality testing and evaluation of pan bread}

Measures of grain hardness, protein content, Zeleny sedimentation values, Farinograph and Extensograph parameters, and pan bread qualities were performed by methods reported in $\mathrm{He}$ et al. [55]. The glutenin macropolymer compositions were measured following Zhang et al. [56].

\section{Statistical analysis}

The SAS statistical package (SAS Institute, Cary, NC) was used for data analysis. All statistical analyses were based on averaged data from two locations.

\section{Additional files}

Additional file 1: Figure S1. Quantitative gel densitometry measurements of B-group proteins in Glu-B3 NILs as determined using NIL ImageJ software program. Data points were normalized with respect to the Aroona value. Figure S2: Sequence alignments of i-type proteins identified in Aroona NILs. Figure S3: Sequence alignments of the B3-530 protein and its allelic variants identified in Aroona NILs. Figure S4: Sequence alignments of the B3-544/593/601/607 proteins identified in Aroona NILs. Figure S5: Sequence alignments of the B3-621/624 and B3688 proteins identified in Aroona NILs. Figure S6: The differential display of B3-688 spots among Aroona-Glu-B3C, B3d and B3h. Spot volume values are expressed in percentages (\%vol) of the total proteome.

Additional file 2: Table S1. F values of one way ANOVA of wheat quality properties by locations and genotypes. Table S2: Comparison of pan bread quality properties among Aroona NILs. Table S3: Comparison of dough properties among Aroona NILs. Table S4: Comparison of glutenin macro-polymer properties among Aroona NILs. Table S5: Composition of glutenin alleles in Aroona and its NILs.

\section{Abbreviations}

2D-PAGE: Two-dimensional gel electrophoresis (IEF $\times$ SDS-PAGE); \%UPP: UPP/ $(\mathrm{UPP}+\mathrm{EPP}) \times 100$; ANOVA: Analysis of variance; CIMMYT: International maize and wheat improvement center; cMs: Centimorgans; DT: Farinograph development time (min); DTT: Dithiothreitol; EA: Energy area $\left(\mathrm{cm}^{2}\right)$; EC: External color; EPP: Extractable glutenin polymeric protein; Ext: Extensograph extensibility (mm); Gli/Glu: Ratio of gliadin to glutenin; GMP: Glutenin macro-polymers; HMW-GS: High-molecular-weight glutenin subunits; HPLC: High-performance liquid chromatography; IC: Inner color; IEF: Isoelectric focusing; kDa: Kilodalton; KP: Kernel protein (\%, 14\% m.b.); LMW-GS: Low-molecular-weight glutenin subunits; LV: Loaf volume $\left(\mathrm{cm}^{3}\right)$; LVS: Loaf volume score; NIL: Near-isogenic lines; ORFs: Open reading frame; PBTS: Pan bread total score; PCR: Polymerase chain reaction; pl: Isoelectric points; RILs: Recombinant inbred lines; Rmax: Extensograph maximum resistance (B.U.); SDS: Sodium dodecyl sulfate; SDS-PAGE: Sodium dodecyl sulphate-polyacrylamide gel electrophoresis; Sha: Shape; Smo: Smoothness; Spr: Springiness; ST: Farinograph stability time (min); Str: Structure; TCA: Trichloroacetic acid; TF: Taste flavor; Tris- $\mathrm{HCl}$ : Tris (hydroxymethyl) aminomethane hydrochloride; UPP: Unextractable glutenin polymeric protein; Wab: Farinograph water absorption (\%); ZSV: Zeleny sedimentation value $(\mathrm{mL})$.

\section{Authors' contributions}

XZ carried out SDS-PAGE, 2D-PAGE and LMW-GS gene marker system analyses and drafted the manuscript. HJ measured glutenin macro-polymers, dough properties and pan bread quality parameters and performed statistical analysis. $Y Z$ participated in the measurement of processing quality parameters. DL participated in the identification of LMW-GS genes and proteins with SDS-PAGE, 2D-PAGE and LMW-GS gene marker system and revised the manuscript. GL participated in the design of the study and quality parameter analysis. XX participated in the design of the study and revised the manuscript. $\mathrm{ZH}$ conceived of the study and participated in its design, coordinated the various research groups, and revised the manuscript. AZ conceived of the study and participated in its design, and revised the manuscript. All authors read and approved the final manuscript.

\section{Financial competing interests}

- In the past five years have you received reimbursements, fees, funding, or salary from an organization that may in any way gain or lose financially from the publication of this manuscript, either now or in the future? Is such an organization financing this manuscript (including the article-processing charge)? If so, please specify. No.

- Do you hold any stocks or shares in an organization that may in any way gain or lose financially from the publication of this manuscript, either now or in the future? If so, please specify.

No.

- Do you hold or are you currently applying for any patents relating to the content of the manuscript? Have you received reimbursements, fees, funding, or salary from an organization that holds or has applied for patents relating to the content of the manuscript? If so, please specify. No.

- Do you have any other financial competing interests? If so, please specify. No.

\section{Non-financial competing interests}

Are there any non-financial competing interests (political, personal, religious, ideological, academic, intellectual, commercial or any other) to declare in relation to this manuscript? If so, please specify. No.

\section{Acknowledgement}

The authors are grateful to Prof. Robert McIntosh, University of Sydney, for reviewing this manuscript. We thank Drs Marie Appelbee and Ken Shepherd, SARDI Grain Quality Research Laboratory, Adelaide, South Australia, for seed of the spring wheat variety Aroona and near-isogenic derivatives. This study was supported by the National Basic Research Program (2009CB118300), the National Science Foundation of China (30830072), International Collaboration Project from Ministry of Agriculture (2011-G3), and China Agriculture Research System (CARS-3-1-3). 


\section{Author details}

'Institute of Crop Science, National Wheat Improvement Center, Chinese Academy of Agricultural Sciences (CAAS), 12 Zhongguancun South Street, Beijing 100081, China. ${ }^{2}$ State Key Laboratory of Plant Cell and Chromosome Engineering, National Center for Plant Gene Research, Institute of Genetics and Developmental Biology, Chinese Academy of Sciences, 1 West Beichen Road, Beijing 100101, China. ${ }^{3}$ Crop Research Institute, Shandong Academy of Agricultural Sciences, Jinan 250100Shandong, China. ${ }^{4}$ International Maize and Wheat Improvement Center (CIMMYT) China Office, c/o CAAS, 12 Zhongguancun South Street, Beijing 100081, China.

Received: 27 July 2012 Accepted: 17 December 2012

Published: 22 December 2012

\section{References}

1. Osborne TB: The vegetable proteins. London: Longmans Green and Co; 1924:154.

2. Wasik RJ, Bushuk W: Sodium dodecyl sulfate-polyacrylamide gel-electrophoresis of reduced glutenin of durum wheats of different spaghetti-making quality. Cereal Chem 1975, 52(3):328-334.

3. Payne PI: Genetics of wheat storage proteins and the effect of allelic variation on bread-making quality. Ann Rev Plant Physiol 1987, 38:141-153.

4. D'Ovidio R, Masci S: The low-molecular-weight glutenin subunits of wheat gluten. J Cereal Sci 2004, 39(3):321-339.

5. Shewry PR, Tatham AS, Barro F, Barcelo P, Lazzeri P: Biotechnology of breadmaking: unraveling and manipulating the multi-protein gluten complex. Nat Biotechnol 1995, 13(11):1185-1190.

6. Gao S, Gu YQ, Wu J, Coleman-Derr D, Huo N, Crossman C, Jia J, Zuo Q, Ren Z, Anderson OD, et al: Rapid evolution and complex structural organization in genomic regions harboring multiple prolamin genes in the polyploid wheat genome. Plant Mol Biol 2007, 65(1-2):189-203.

7. Pogna NE, Autran JC, Mellini F, Lafiandra D, Feillet P: Chromosome $1 B$ encoded gliadins and glutenin subunits in durum wheat: genetics and relationship to gluten strength. J Cereal Sci 1990, 11(1):15-34.

8. Ruiz M, Carrillo JM: Linkage relationships between prolamin genes on chromosome $1 A$ and chromosome $1 B$ of durum wheat. Theor Appl Genet 1993, 87(3):353-360.

9. Cassidy BG, Dvorak J, Anderson OD: The wheat low-molecular-weight glutenin genes: characterization of six new genes and progress in understanding gene family structure. Theor Appl Genet 1998, 96(6-7):743-750.

10. Huang $X Q$, Cloutier S: Molecular characterization and genomic organization of low molecular weight glutenin subunit genes at the Glu-3 loci in hexaploid wheat (Triticum aestivum L.). Theor Appl Genet 2008, 116(7):953-966.

11. Singh NK, Shepherd KW, Cornish GB: A simplified SDS-PAGE procedure for separating LMW subunits of glutenin. J Cereal Sci 1991, 14(3):203-208.

12. Liu L, Ikeda TM, Branlard G, Pena RJ, Rogers WJ, Lerner SE, Kolman MA, Xia $X$, Wang L, Ma W, et al: Comparison of low molecular weight glutenin subunits identified by SDS-PAGE, 2-DE. MALDI-TOF-MS and PCR in common wheat. BMC Plant Biol 2010, 10:124.

13. Wang LH, Li GY, Pena RJ, Xia XC, He ZH: Development of STS markers and establishment of multiplex PCR for Glu-A3 alleles in common wheat (Triticum aestivum L.). J Cereal Sci 2010, 51(3):305-312.

14. Wang LH, Zhao XL, He ZH, Ma W, Appels R, Pena RJ, Xia XC: Characterization of low-molecular-weight glutenin subunit Glu-B3 genes and development of STS markers in common wheat (Triticum aestivum L.). Theor Appl Genet 2009, 118(3):525-539.

15. Zhang W, Gianibelli MC, Rampling LR, Gale KR: Characterisation and marker development for low molecular weight glutenin genes from Glu-A3 alleles of bread wheat (Triticum aestivum. L). Theor Appl Genet 2004, 108(7):1409-1419.

16. Zhao XL, Xia XC, He ZH, Gale KR, Lei ZS, Appels R, Ma W: Characterization of three low-molecular-weight Glu-D3 subunit genes in common wheat. Theor Appl Genet 2006, 113(7):1247-1259.

17. Zhao XL, Xia XC, He ZH, Lei ZS, Appels R, Yang Y, Sun QX, Ma W: Novel DNA variations to characterize low molecular weight glutenin Glu-D3 genes and develop STS markers in Common Wheat. Theor Appl Genet 2007, 114(3):451-460.

18. Dong LL, Zhang XF, Liu DC, Fan HJ, Sun JZ, Zhang ZJ, Qin HJ, Li B, Hao ST, Li ZS, et al: New insights into the organization, recombination, expression and functional mechanism of low molecular weight glutenin subunit genes in bread wheat. PLoS One 2010, 5(10):e13548. doi:doi:10.1371/journal.pone.0013548.

19. Ikeda TM, Araki E, Fujita Y, Yano H: Characterization of low-molecularweight glutenin subunit genes and their protein products in common wheats. Theor Appl Genet 2006, 112(2):327-334.

20. Ikeda TM, Nagamine T, Fukuoka H, Yano H: Identification of new low-molecular-weight glutenin subunit genes in wheat. Theor Appl Genet 2002, 104(4):680-687.

21. Zhang XF, Liu DC, Jiang W, Guo XL, Yang WL, Sun JZ, Ling HQ, Zhang AM: PCR-based isolation and identification of full-length low-molecular-weight glutenin subunit genes in bread wheat (Triticum aestivum L.). Theor App/ Genet 2011, 123(8):1293-1305.

22. Zhang XF, Liu DC, Yang WL, Liu KF, Sun JZ, Guo XL, Li YW, Wang DW, Ling $H Q$, Zhang AM: Development of a new marker system for identifying the complex members of the low-molecular-weight glutenin subunit gene family in bread wheat (Triticum aestivum L.). Theor Appl Genet 2011, 122(8):1503-1516.

23. Payne $\mathrm{Pl}$, Nightingale MA, Krattiger AF, Holt LM: The relationship between HMW glutenin subunit composition and the bread-making quality of British grown wheat varieties. J Sci Food Agr 1987, 40(1):51-65.

24. Branlard G, Dardevet M, Saccomano R, Lagoutte F, Gourdon J: Genetic diversity of wheat storage proteins and bread wheat quality. Euphytica 2001, 119(1-2):59-67.

25. Brett GM, Mills ENC, Tatham AS, Fido RJ, Shewry PR, Morgan MRA: Immunochemical identification of LMW subunits of glutenin associated with bread-making quality of wheat flours. Theor Appl Genet 1993, 86(4):442-448.

26. Flaete NES, Uhlen AK: Association between allelic variation at the combined Gli-1, Glu-3 loci and protein quality in common wheat (Triticum aestivum L.). J Cereal Sci 2003, 37(2):129-137.

27. Gupta RB, Bekes F, Wrigley CW: Prediction of physical dough properties from glutenin subunit composition in bread wheats - correlation studies. Cereal Chem 1991, 68(4):328-333.

28. Gupta RB, Paul JG, Cornish GB, Palmer GA, Bekes F, Rathjen AJ: Allelic variation at glutenin subunit and gliadin loci, Glu-1, Glu-3 and Gli-1, of Common Wheat. 1. Its additive and interaction effects on dough properties. J Cereal Sci 1994, 19(1):9-17.

29. Luo C, Griffin WB, Branlard G, MCNeil DL: Comparison of low- and high molecular-weight wheat glutenin allele effects on flour quality. Theor App/ Genet 2001, 102(6-7):1088-1098.

30. Nietotaladriz MT, Perretant MR, Rousset M: Effect of gliadins and HMW and LMW subunits of glutenin on dough properties in the F-6 recombinant inbred lines from a bread wheat cross. Theor Appl Genet 1994, 88(1):81-88.

31. Nagamine T, Kai Y, Takayama T, Yanagisawa T, Taya S: Allelic variation at the G/u-1 and Glu-3 loci in southern Japanese wheats, and its effects on gluten properties. J Cereal Sci 2000, 32(2):129-135.

32. Bekes F, Kemeny S, Morell M: An integrated approach to predicting endproduct quality of wheat. Eur J Agron 2006, 25(2):155-162.

33. Eagles HA, Hollamby GJ, Gororo NN, Eastwood RF: Estimation and utilisation of glutenin gene effects from the analysis of unbalanced data from wheat breeding programs. Aust J Agr Res 2002, 53(4):367-377.

34. Cane K, Sharp PJ, Eagles HA, Eastwood RF, Hollamby GJ, Kuchel H, Lu M, Martin PJ: The effects on grain quality traits of a grain serpin protein and the VPM1 segment in southern Australian wheat breeding. Aust J Agr Res 2008, 59(10):883-890.

35. Zheng SS, Byrne PF, Bai GH, Shan XY, Reid SD, Haley SD, Seabourn BW: Association analysis reveals effects of wheat glutenin alleles and rye translocations on dough-mixing properties. J Cereal Sci 2009, 50(2):283-290.

36. Dupont FM, Vensel WH, Tanaka CK, Hurkman WJ, Altenbach SB: Deciphering the complexities of the wheat flour proteome using quantitative twodimensional electrophoresis, three proteases and tandem mass spectrometry. Proteome Sci 2011, 9:10. doi:10.1186/1477-5956-9-10.

37. Gupta RB, Singh NK, Shepherd KW: The cumulative effect of allelic variation in LMW and HMW glutenin subunits on dough properties in the progeny of two bread wheats. Theor Appl Genet 1989, 77(1):57-64.

38. Park CS, Kang C-S, Jeung J-U, Woo S-H: Influence of allelic variations in glutenin on the quality of pan bread and white salted noodles made from Korean wheat cultivars. Euphytica 2011, 180(2):235-250.

39. Gupta RB, Macritchie F: Allelic variation at glutenin subunit and gliadin loci, Glu-1, Glu-3 and Gli-1 of Common Wheats. 2. Biochemical basis of the allelic effects on dough properties. J Cereal Sci 1994, 19(1):19-29. 
40. Ito M, Fushie S, Maruyama-Funatsuki W, Ikeda TM, Nishio Z, Nagasawa K, Tabiki T, Yamauchi H: Effect of allelic variation in three glutenin loci on dough properties and bread-making qualities of winter wheat. Breeding Sci 2011, 61(3):281-287.

41. Liu L, He ZH, Yan J, Zhang Y, Xia XC, Pena RJ: Allelic variation at the Glu-1 and Glu-3 loci, presence of the 1B.1R translocation, and their effects on mixographic properties in Chinese bread wheats. Euphytica 2005, 142(3):197-204

42. Meng XG, Xie F, Shang XW, An LZ: Association between allelic variations at the Glu-3 loci and wheat quality traits with Lanzhou Alkaline Stretched Noodles quality in northwest China spring wheats. Cereal Res Commun 2007, 35(1):109-118.

43. Bekes F, Gras PW, Anderssen RS, Appels R: Quality traits of wheat determined by small-scale dough testing methods. Aust J Agr Res 2001, 52(11-12):1325-1338.

44. Ma W, Appels R, Bekes F, Larroque O, Morell MK, Gale KR: Genetic characterisation of dough rheological properties in a wheat doubled haploid population: additive genetic effects and epistatic interactions. Theor Appl Genet 2005, 111(3):410-422.

45. Martinez-Cruz E, Espitia-Rangel E, Villasenor-Mir HE, Molina-Galan JD, Benitez-Riquelme I, Santacruz-Varela A, Pena-Bautista RJ: Dough rheology of wheat recombinant lines in relation to allelic variants of Glu-1 and Glu-3 loci. Cereal Res Commun 2011, 39(3):386-393.

46. Li Y, Zhou R, Branlard G, Jia J: Development of introgression lines with 18 alleles of glutenin subunits and evaluation of the effects of various alleles on quality related traits in wheat (Triticum aestivum L.). J Cereal Sci 2010, 51(1):127-133.

47. Tabiki T, Ikeguchi S, Ikeda TM: Effects of high-molecular-weight and low-molecular-weight glutenin subunit alleles on common wheat flour quality. Breeding Sci 2006, 56(2):131-136.

48. Liu L, He ZH, Ma WJ, Liu JJ, Xia XC, Pena RJ: Allelic variation at the Glu-D3 locus in Chinese bread wheat and effects on dough properties, pan bread and noodle qualities. Cereal Res Commun 2009, 37(1):57-64.

49. Gobaa S, Bancel E, Branlard G, Kleijer G, Stamp P: Proteomic analysis of wheat recombinant inbred lines: Variations in prolamin and dough rheology. J Cereal Sci 2008, 47(3):610-619.

50. Popineau Y, Cornec M, Lefebvre J, Marchylo B: Influence of high M(r) glutenin subunits on glutenin polymers and rheological properties of glutens and gluten subfractions of near-isogenic lines of wheat Sicco. J Cereal Sci 1994, 19(3):231-241.

51. Saghai-Maroof MA, Soliman KM, Jorgensen RA, Allard RW: Ribosomal DNA spacer-length polymorphisms in barley: mendelian inheritance, chromosomal location, and population dynamics. Proc Nat Acad Sci USA 1984, 81(24):8014-8018.

52. Kumar S, Nei M, Dudley J, Tamura K: MEGA: a biologist-centric software for evolutionary analysis of DNA and protein sequences. Brief Bioinform 2008, 9(4):299-306.

53. Sambrook J, Russell DW: Molecular cloning: a laboratory manual. New York: Cold Spring Harbor Laboratory Press; 2001.

54. Wang W, Vignani $R$, Scali M, Cresti M: A universal and rapid protocol for protein extraction from recalcitrant plant tissues for proteomic analysis. Electrophoresis 2006, 27(13):2782-2786.

55. He ZH, Liu L, Xia XC, Liu JJ, Pena RJ: Composition of HMW and LMW glutenin subunits and their effects on dough properties, pan bread, and noodle quality of Chinese bread wheats. Cereal Chem 2005, 82(4):345-350.

56. Zhang PP, He ZH, Zhang Y, Xia XC, Chen DS, Zhang Y: Association between \% SDS-unextractable polymeric protein (\%UPP) and end-use quality in Chinese bread wheat cultivars. Cereal Chem 2008, 85(5):696-700.

doi:10.1186/1471-2229-12-243

Cite this article as: Zhang et al.: Composition and functional analysis of low-molecular-weight glutenin alleles with Aroona near-isogenic lines of bread wheat. BMC Plant Biology 2012 12:243.

\section{Submit your next manuscript to BioMed Central and take full advantage of:}

- Convenient online submission

- Thorough peer review

- No space constraints or color figure charges

- Immediate publication on acceptance

- Inclusion in PubMed, CAS, Scopus and Google Scholar

- Research which is freely available for redistribution

Submit your manuscript at www.biomedcentral.com/submit 